\title{
Hybrid Green Bionanocomposites of Bio-based Poly(butylene succinate) Reinforced with Pyrolyzed Perennial Grass Microparticles and Graphene Nanoplatelets
}

Connor J. Cooper 1,2, Mohamed A. Abdelwahab ${ }^{1,3}$, Amar K. Mohanty 1, 2, *, Manjusri Misra 1, 2, * ${ }^{1}$ School of Engineering, Thornbrough Building, University of Guelph, Guelph, N1G 2W1, Ontario, Canada

${ }^{2}$ Bioproducts Discovery and Development Centre, Department of Plant Agriculture, Crop Science Building, University of Guelph, Guelph, N1G 2W1, Ontario, Canada

${ }^{3}$ Department of Chemistry, Tanta University, Tanta, 31527, Egypt

* Correspondence to M. Misra, Email: mmisra@uoguelph.ca and Amar K. Mohanty. Email: mohanty@uoguelph.ca

Figure S1: Zero shear viscosity (left) and melt flow index (right). Where (A) BioPBS, (B) BioPBS/BC (90/10), (C) BioPBS/BC (85/15), (D) BioPBS/BC (80/20), (E) BioPBS/BC (75/25), (F-G) BioPBS/BC/GnP (MB) (75/24/1) and (75/20/5), respectively, and (H-I) BioPBS/BC/GnP (DC) $(75 / 24 / 1)$ and $(75 / 20 / 5)$, respectively.

Table S1: Thermal data of the neat polymer and composite samples.

Table S2: $2^{2}$ analysis of variance of impact strength on $\mathrm{GnP}$ content and processing methods, alpha -0.05 .

Table S3: $2^{2}$ analysis of variance of tensile strength on of $\mathrm{GnP}$ content and processing methods, alpha -0.05 .

Table S4: Two-Sample t test, assuming unequal variances, of CLTE and HDT measurements, alpha -0.05 


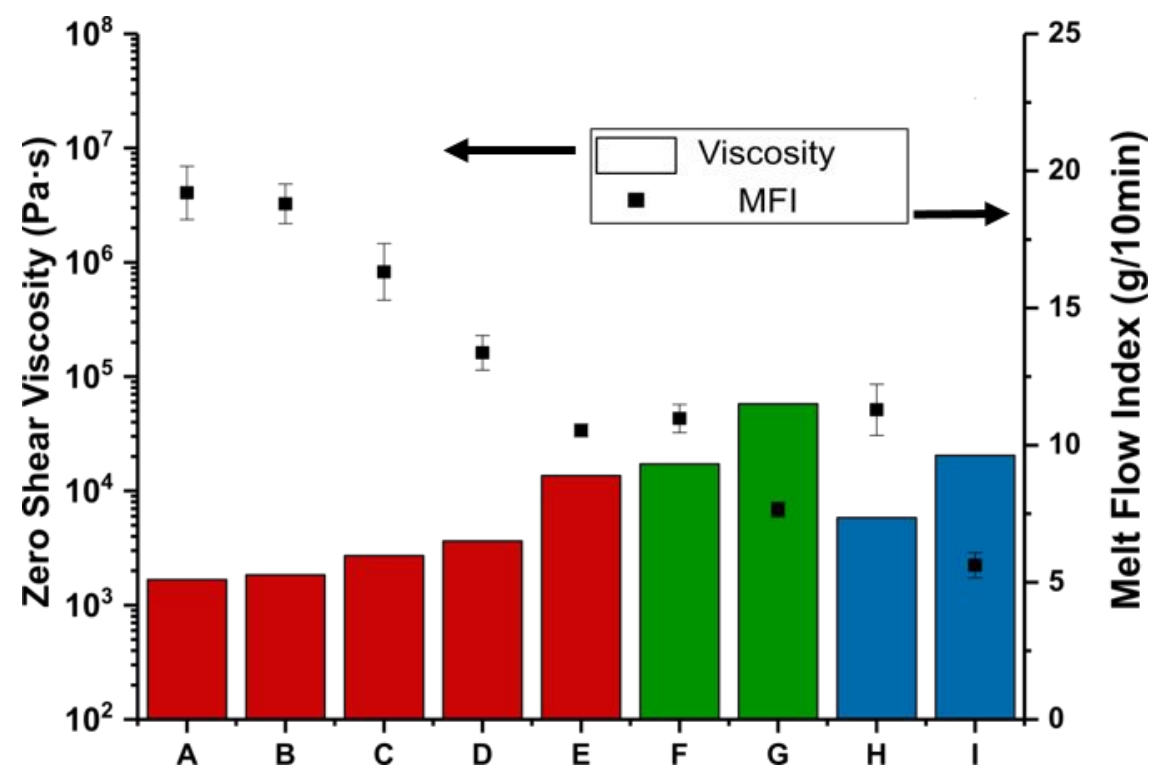

Figure S1. Zero shear viscosity (left) and melt flow index (right). Where (A) BioPBS, (B) BioPBS/BC (90/10), (C) BioPBS/BC (85/15), (D) BioPBS/BC (80/20), (E) BioPBS/BC (75/25), (F-G) BioPBS/BC/GnP (MB) (75/24/1) and (75/20/5), respectively, and (H-I) BioPBS/BC/GnP (DC) (75/24/1) and (75/20/5), respectively.

Table S1. Thermal data of the neat polymer and composite samples.

\begin{tabular}{|c|c|c|c|c|c|}
\hline Samples & $\begin{array}{c}T_{m} \\
\left({ }^{\circ} \mathrm{C}\right)\end{array}$ & $\begin{array}{l}\Delta H_{m} \\
(\mathrm{~J} / \mathrm{g})\end{array}$ & $\begin{array}{c}T_{c} \\
\left({ }^{\circ} \mathrm{C}\right)\end{array}$ & $\begin{array}{l}\Delta H_{c} \\
(\mathrm{~J} / \mathrm{g})\end{array}$ & Crystallinity (\%) \\
\hline BioPBS & $115.7 \pm 0.03$ & $70.5 \pm 1.63$ & $91.2 \pm 0.05$ & $69.2 \pm 0.43$ & 34.60 \\
\hline BioPBS/10BC & $115.2 \pm 0.16$ & $57.5 \pm 0.44$ & $89.4 \pm 0.05$ & $62.8 \pm 0.24$ & 28.26 \\
\hline BioPBS/15BC & $115.8 \pm 0.01$ & $50.9 \pm 0.39$ & $89.8 \pm 0.20$ & $55.5 \pm 0.76$ & 23.61 \\
\hline BioPBS/20BC & $115.5 \pm 0.25$ & $48.1 \pm 0.54$ & $88.4 \pm 0.22$ & $52.1 \pm 0.87$ & 20.83 \\
\hline BioPBS/25BC & $115.6 \pm 0.40$ & $44.0 \pm 0.08$ & $87.9 \pm 0.29$ & $48.6 \pm 1.0$ & 18.23 \\
\hline $\begin{array}{l}\text { BioPBS/24BC/1GnP } \\
\text { - master batch - }\end{array}$ & $114.8 \pm 0.57$ & $54.3 \pm 3.27$ & $96.4 \pm 0.11$ & $51.1 \pm 1.1$ & 19.15 \\
\hline $\begin{array}{l}\text { BioPBS } / 20 B C / 5 G n P \\
\text { - master batch - }\end{array}$ & $114.0 \pm 0.29$ & $54.3 \pm 1.53$ & $98.8 \pm 0.12$ & $49.5 \pm 0.5$ & 18.56 \\
\hline $\begin{array}{c}\text { BioPBS/24BC/1GnP } \\
\text { - direct - }\end{array}$ & $114.9 \pm 0.04$ & $51.2 \pm 0.08$ & $95.9 \pm 0.11$ & $50.6 \pm 0.76$ & 18.98 \\
\hline $\begin{array}{c}\text { BioPBS } / 20 B C / 5 G n P \\
\text { - direct - }\end{array}$ & $115.3 \pm 0.85$ & $52.4 \pm 0.55$ & $98.2 \pm 0.14$ & $51.4 \pm 1.3$ & 19.27 \\
\hline $\begin{array}{l}\text { BioPBS/25GnP } \\
\text { - master batch - }\end{array}$ & $114.6 \pm 1.00$ & $51.9 \pm 0.47$ & $99.3 \pm 0.33$ & $52.0 \pm 0.24$ & 19.51 \\
\hline
\end{tabular}


Table S2: $2^{2}$ analysis of variance of impact strength on GnP content and processing methods, alpha - 0.05.

$2^{2}$ ANOVA with replicates - Impact Strength

\begin{tabular}{lcrcccc}
\hline \multicolumn{1}{c}{ Source of Variation } & SS & $d f$ & $M S$ & $F$ & P-value & F crit \\
\hline Process method & 415.9637 & 1 & 415.9637 & 255.9819 & $2.33 \mathrm{E}-07$ & 5.317655 \\
Amount of GnP & 123.7198 & 1 & 123.7198 & 76.13656 & $2.33 \mathrm{E}-05$ & 5.317655 \\
Interaction & 70.15585 & 1 & 70.15585 & 43.17356 & 0.000175 & 5.317655 \\
Within & 12.99978 & 8 & 1.624973 & & & \\
& & & & & & \\
Total & 622.8391 & 11 & & & & \\
\hline
\end{tabular}

Table S3: $2^{2}$ analysis of variance of tensile strength on of GnP content and processing methods, alpha -0.05 .

$2^{2}$ ANOVA with replicates - Tensile Strength

\begin{tabular}{lrrrrrc}
\hline \multicolumn{1}{c}{ Source of Variation } & \multicolumn{1}{c}{ SS } & \multicolumn{1}{c}{ df } & \multicolumn{1}{c}{ MS } & \multicolumn{1}{c}{ F } & P-value & \multicolumn{1}{c}{ F crit } \\
\hline Process method & 11.1005 & 1 & 11.1005 & 11.17031 & 0.004135 & 4.493998 \\
Amount of GnP & 11.7045 & 1 & 11.7045 & 11.77811 & 0.003422 & 4.493998 \\
Interaction & 0.0405 & 1 & 0.0405 & 0.040755 & 0.842557 & 4.493998 \\
Within & 15.9 & 16 & 0.99375 & & & \\
& & & & & & \\
Total & 38.7455 & 19 & & & & \\
\hline
\end{tabular}

Table S4: Two-Sample t test, assuming unequal variances, of CLTE and HDT measurements, alpha - 0.05.

\begin{tabular}{|c|c|c|c|c|c|c|c|c|}
\hline & $\begin{array}{c}\text { BioPBS/ } \\
\text { 10BC }\end{array}$ & $\begin{array}{c}\text { BioPBS/ } \\
15 B C\end{array}$ & $\begin{array}{c}\text { BioPBS/ } \\
20 B C\end{array}$ & $\begin{array}{c}\text { BioPBS/ } \\
25 B C\end{array}$ & $\begin{array}{c}\text { BioPBS/ } \\
24 B C / \\
1 G n P \\
(M B)\end{array}$ & $\begin{array}{c}\text { BioPBS/ } \\
20 B C / \\
5 G n P \\
(M B)\end{array}$ & $\begin{array}{c}\text { BioPBS/ } \\
24 B C / \\
1 G n P \\
(D C)\end{array}$ & $\begin{array}{c}\text { BioPBS/ } \\
20 B C / \\
5 G n P \\
(D C)\end{array}$ \\
\hline $\begin{array}{c}\text { CLTE } \\
\text { t stat }\end{array}$ & -0.147 & 9.66 & 4.60 & 8.71 & 10.4 & 4.12 & 9.85 & 9.88 \\
\hline $\begin{array}{c}\text { CLTE } \\
\text { t critical }\end{array}$ & 12.7 & 12.7 & 12.7 & 4.30 & 12.7 & 12.7 & 12.7 & 12.7 \\
\hline $\begin{array}{l}\text { HDT } \\
\text { t stat }\end{array}$ & -1.97 & -6.21 & -6.77 & -12.6 & -8.88 & -7.46 & -7.57 & -8.98 \\
\hline $\begin{array}{c}\text { HDT } \\
\text { t critical }\end{array}$ & 4.30 & 12.7 & 12.7 & 12.7 & 12.7 & 12.7 & 4.30 & 12.7 \\
\hline
\end{tabular}

